\title{
Experimental study of through the wall imaging for the detection of vital life signs using SFWR
}

Pardhu Thottempudi, Vijay Kumar

School of Electronics Engineering, VIT University, Vellore, India

\begin{tabular}{l} 
Article Info \\
\hline Article history: \\
Received Apr 28, 2021 \\
Revised Aug 30, 2021 \\
Accepted Sep 6, 2021 \\
\hline
\end{tabular}

Keywords:

Detection

RADAR

SFCWR

TWI

VNA

\begin{abstract}
Now a day's defence applications associated to novel, army and military war fields are required wall imaging discrimination. As of now many wallimaging techniques are designed but didn't identify the vital signs behind walls with accurate working. Therefore, a novel advance wall image tracking method is required identification of human target. An experimental study on through the wallimaging (TWI) to detect the life signs using sweep frequency continuous wave radar (SFCWR) is explained in this paper. The proposed system consists of agilent vector network analyzer (VNA) (Agilent E5071B ENA), horn antenna and a computer. The information of heart beat and the breathing can be a shift identification routine was used to collect information from the back scattering electric current. The outcomes of the procedure give the information of heart beat and breathing signs of real human being.
\end{abstract}

This is an open access article under the CC BY-SA license.

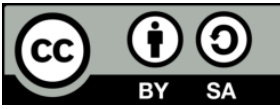

\section{Corresponding Author:}

Pardhu Thottempudi

School of Electronics Engineering (SENSE)

VIT University

Vellore, Tamil Nadu, India

Email: pthottempudi2020@gmail.com

\section{INTRODUCTION}

Through-the-wall radar (TWR) is a new tool that can be used to track and locate unseen items hidden behind walls. It has a wide range of uses, including victim identification in evacuation and hostage situations, as well as human localization in anti-terrorist actions and life detection in earthquake debris [1]. Microwave doppler radars [2] have recently been designed for remote sensing of pulse and breathing. The incidence, phase, intensity, and delivery the time it takes for a live human body to return a signal can varyall be affected by pulse and breathing movements. Instead to the thickness and electrical assets of the wall content, these responses can be significantly reduced [2]. As a result, obtaining accurate radar-based identification of pulse or breathing is generally regarded as a difficult challenge. In this study, they used a phased frequency rf signals radar system with a large fractional bandwidth (50 percent) [3]. As a result, shift detection (CD) algorithms will identify human life signals hidden behind walls. Breathing is a repetitive behaviour that occurs at regular intervals. The subtraction of two consecutive data sets yields the discrepancy signals. On 2D time-varying range profile figure, the difference signal appears as the breathing's periodic behaviour. Pardhu and Kumar [3] clearly explained about the interfacing of radar to vector network analyzer (VNA) and receiving of echo [4]. Li et al. [5] explained about human identification using ultra-wideband (UWB) radar with low frequency. Where as we have done the identification using sweep frequency continuous wave radar (SFCWR) which operates at high frequency and also in multiple clutter situation. 


\section{RADAR SYSTEM DESCRIPTION}

We used a monostatic setup in the presence of a insubstantial wall with a thickness of $30 \mathrm{~cm}$ in the tests. A C-band pyramidal array antenna was used as a monitor, and the Agilent E5071B ENA vector system analyser (VSA) frequency was changed from 3 to $5 \mathrm{GHz}$ for 201 linearly treaded stages. Figure 1 depicts the experimental radar setup. Radar specifications that we are using in this experiment is given in in Table 1.

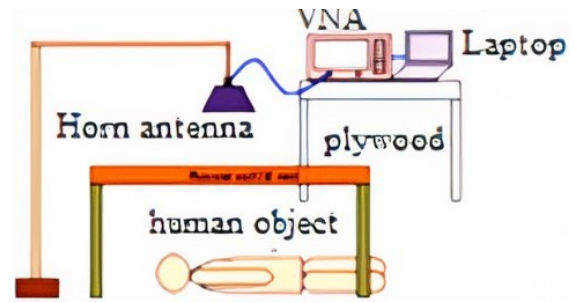

(a)

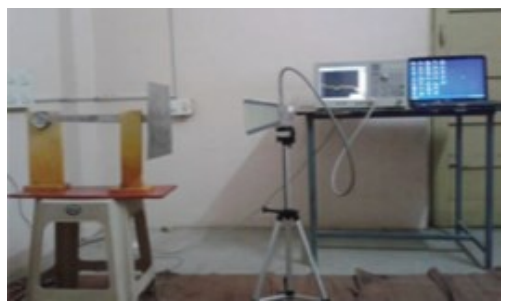

(b)

Figure 1. These figures are (a) experimental setup and (b) experimental setup model

Table 1. Radar parameters and specifications

\begin{tabular}{cll}
\hline S.No & \multicolumn{1}{c}{$\begin{array}{c}\text { Radar parameters as well as specifications } \\
\text { Parameter }\end{array}$} & \multicolumn{1}{c}{ Specification } \\
\hline 1 & Frequency Range of Operation & $1 \mathrm{GHz}$ to $3 \mathrm{GHz}$ \\
2 & Power that is radiated & $0 \mathrm{dBm}$ \\
3 & Step Size of Frequency & $10 \mathrm{MHz}$ \\
4 & Amount of traces & 1,024 \\
5 & Swept Time & $0.024881 \mathrm{sec}$ \\
6 & Range Resolution in Air & $7.5 \mathrm{~cm}$ \\
\hline
\end{tabular}

\section{METHODOLOGY}

Let transmitter and receiver of UWB radar are placed at $x_{t}, x_{r}$, while nominal chest position is at $x_{c}$ and abdomen position is at $x_{a}$ of stationary human target. The receiver signal $d(t)$ is obtained after abdomen and chest reflections of human objective positioned at $d_{0}$ is known by $d(t)$ and presented in Figure 2 [6]-[10].

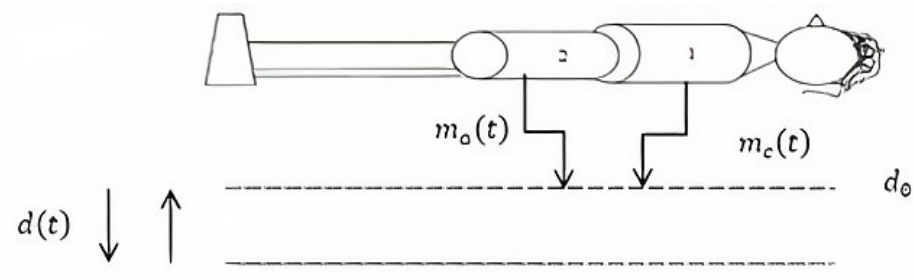

Figure 2. Breathing model of human target

$$
\begin{aligned}
& d(t)=d_{0}+m_{c}(t)+m_{a}(t) \\
& =d_{0}+\sum_{i=1}^{M} c_{i} \sin \left(2 \pi i f_{h} t\right)+\sum_{j=1}^{N} a_{j} \sin \left(2 \pi i f_{b} t\right)
\end{aligned}
$$

Where $c_{i}, a_{i}$ represents harmonic components of displacement of chest and abdomen, $f_{b}$ represents frequency of breathing $\&_{M, N}$ represents count of significant components of abdomen and chest displacement. Displacement of heart is ignored as radar doesn't had sufficient resolution to detect heart motion. The output of the correlator can be represented as the variation due to abdomen and chest, the amount of the channel's multiple answers [11]-[15]:

$$
r(t, \tau)=\sum_{i} A_{i} p\left(\tau-\tau_{i}\right)+A p\left(\tau-\tau_{d}(t)\right)
$$

where $p(t)$ represents correlator output. $A_{i}$ Givescorrelator output related to the $i^{\text {th }}$ static multipath component, $\tau_{i}$ represents delay. $A$ Represents the correlator output related to reflections from body. $\tau_{d}(t)$ is 
the time delay related with abdominal \& chest movements and represented as a total of flight time $\tau_{0}$ and two delays are represented as (4).

$$
\tau_{d}(t)=\tau_{0}+\sum_{i}^{M} \tau_{i} \sin \left(2 \pi i f_{b} t\right)+\sum_{j}^{N} \mu_{j} \sin \left(2 \pi j f_{b} t\right)
$$

Where $\tau_{0}=\frac{2 d_{0}}{c}=\tau_{i}=\frac{2 c_{i}}{c} \mu_{j}=\frac{2 a_{j}}{c}$ and $c$ is light's maximum speed. The experimentedcorrelator in slow time, output is achieved in distinct instants. $t=k T_{s}, k=1, \ldots ., K$ at discrete fast sample time $\tau=l T_{f}$, $l=1, \ldots \ldots, L$. As the static environment was considered static clutter component appeared as dendritic cells (DC) component and removed by pre-processing algorithm [4]. Let $y(t, \tau)$ be the uncluttered signal i.e it contains the information related to only abdomen and chest movement and is given by (5).

$$
y(t, \tau)=A p\left(\tau-\tau_{0}-\sum_{i=1}^{M} \tau_{i} \sin \left(2 \pi \dot{r} f_{b} t\right)-\sum_{j=1}^{N} \mu_{j} \sin \left(2 \pi f_{b} t\right)\right.
$$

Figures 3(a) and 3(b) shows chest and abdomen alive signals respectively. Figure 3(b) shows the displacements by abdomen cannot be ignored in all UWB radar human detection models [16]-[18]. Figure 4 show the densityfunctional theory (DFT) of recorded signals. It was observed in DFT of abdomen breathing signal that multiple frequency harmonics are present. This designates that the displacements of chest and abdomen are not sinusoidal. Let us consider that the movement of chest is sinusoidal and abdominal movement is semi sinusoidal [19]-[23]. We consider, the movement of chest is sinusoidal (M=1) and the abdominal movement possess the first harmonic (fundamental frequency) and the $2^{\text {nd }}$ harmonic then (5) becomes.

$$
y(t, \tau)=A p\left(\tau-\tau_{0}-\left(\tau_{1}+\mu_{1}\right) \cdot \sin \left(2 \pi_{b} t\right)-\mu_{2} \sin \left(4 \pi f_{b} t\right)\right)
$$

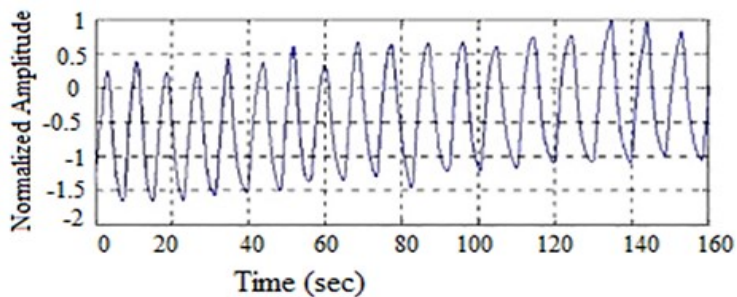

(a)

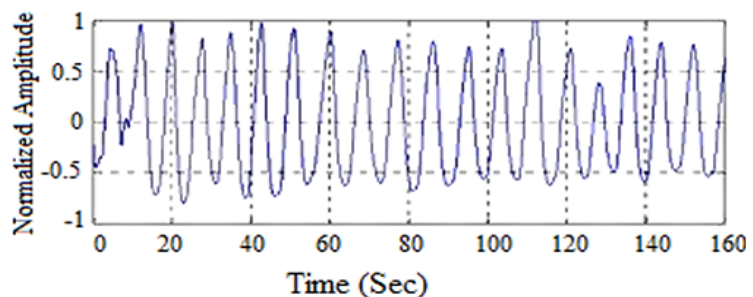

(b)

Figure 3. These figures are (a) chest breathing signal and (b) abdomen breathing signal

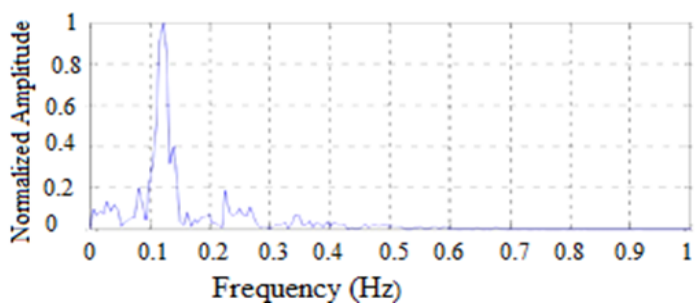

Figure 4. DFT of abdomen breathing signal

The Fourier transform of $y(t, \tau)$ along the slow time can be represented as (7).

$$
Y(f, z)-\int_{-x}^{+\pi}(t, \tau) e^{-2+t} d t
$$

From 2-D Fourier transform (7) can be:

$$
Y(f, \tau)=\int_{-\infty}^{+\pi} Y(f, v) e^{/ 2 \pi t} d v
$$

The 2-D Fourier Transform $Y(f, v)$ can be written as: 


$$
Y(f, v)-A\left(P(v) e^{-12 m t_{1}} \int\left(S_{1} S_{2} S_{5}\right) e^{-12 m} d t\right)
$$

where $P(v)$ represents in the short time of the correlated Fourier transform output:

$$
\begin{aligned}
& S_{1}=\sum_{k=-\infty}^{+\infty} j_{k}\left(\beta_{\tau_{1}} v\right) e^{-j 2 \pi k f t t} \\
& S_{2}=\sum_{l=-\infty}^{+\infty} j_{l}\left(\beta_{\mu_{1}} v\right) e^{-j 2 \pi f_{b} t} \\
& S_{3}=\sum_{m=-\infty}^{+\infty} j_{m}\left(\beta_{\mu_{2}} v\right) e^{-j 2 \pi m\left(2 f_{b}\right) t}
\end{aligned}
$$

where $\beta_{x}=2 \pi x$ and $\sum_{p=-\infty}^{+\infty} J_{p}(z) e^{-j 2 \pi p f t}=e^{-j \sin (2 \pi f t)}$ is Bessel Functions of order $\mathrm{n}$. After simplification it is shown that at $\tau=\tau_{0}$ :

$$
Y\left(f, \tau_{0}\right)=A \sum_{k] \cdot n=-\infty}^{+\infty} C_{k l m} \delta\left(f-(k+l) f_{b}-m\left(2 f_{b}\right)\right)
$$

where:

$$
C_{k l m}=\int_{-\infty}^{+\infty} P(v) j_{k}\left(\beta_{\tau_{1}} v\right) j_{l}\left(\beta_{\mu_{1}} v\right) j_{m}\left(\beta_{\mu_{2}} v\right) d v
$$

Furthermore $C_{k l m}$ can be evaluated using mean value theorem as:

$$
C_{k l m} \approx \Delta f \cdot j_{k}\left(\beta_{\tau_{1}} f_{c}\right) j_{l}\left(\beta_{\mu_{c}} f_{c}\right) j_{m}\left(\beta_{\mu_{2}} f_{c}\right) P\left(f_{c}\right)
$$

The $\Delta f$ is pulse bandwidth. In (15) states that the slow time spectrum contains spectral lines at frequencies of harmonics for breathing frequency as it is discrete.

\section{RESULTS AND DISCUSSION}

A twenty six-year-old guy remained motionless in the space behind the wall first study. A naturally breathing man's reaction was recorded over a 20 -second cycle when the man was standing still. The fact that a chest of human moves produces differing radar proceeds with various back-scattering amplitudes is well established. A dimensional model was created by removing the scene's sequential range profiles in order to plot this shift in the setting. Static artefacts. The radar images displayed in Figure 5 are the product of this shift detection process. The breathing man's time-varying differential range-profiles are then sequentially shown to create a 2D diagram, as seen in Figure 5(a). For all time instants in the snapshot, changes across the $30 \mathrm{~cm}$ range distance can be easily identified [24], [25].

The answer at $30 \mathrm{~cm}$ range is depicted in Figure 5(b) in relation to the observed time period. The figure shows a total of 7 breathings (over a 16-second period), which is compatible with nominal breathing values for a safe person. The monitoring of heartbeats was the subject of the second study. For this reason, the individual stood still for 16 seconds just behind the wall, keeping his breath.

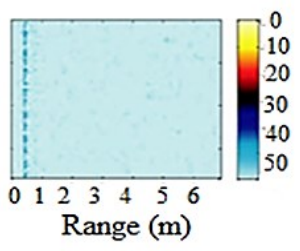

(a)

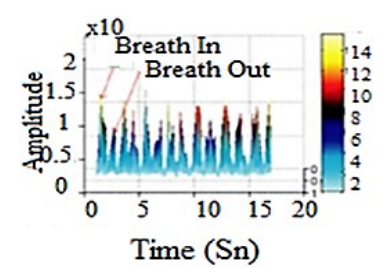

(b)

Figure 5. Radar objects for breathing people: (a) a 2D picture generated by sequentially viewing varianceprofiles-of-ranges and (b) a slice of the resulting copy corresponding to the $30 \mathrm{~cm}$ range

The experimental set-up and parameters remained unchanged from the previous experiment. Figure 6 shows the radar photos that resulted. The only difference in the scene was his pounding heart, so the individual was not able to breathe (and therefore his chest was at rest). As opposed to the breathing experiment, the time-varying range-profiles with poorer reflection were observed and plotted as a twodimensional radar picture in Figure 6(a). Changes in the picture at a $50 \mathrm{~dB}$ amplitude level can be seen at 
$30 \mathrm{~cm}$ spectrum diameter. The plot in Figure 6 is the product of scheming the answer at $30 \mathrm{~cm}$ range space against the practical time period (b). The peaks in this graph refer to the person's heartbeats. A total of 18 peaks were found, corresponding to 64 pulses per minute. This finding is compatible with a balanced man's nominal heart rate of 60 to 80 beats per minute.

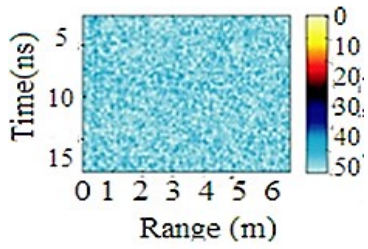

(a)

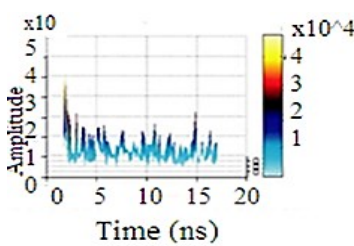

(b)

Figure 6. Heartbeat radar portraits: (a) a 2D picture generated by sequentially viewing the sequential profilesof-ranges and (b) a slice of the resulting copy corresponding to the 30 centi meterset of possibilities

\section{CONCLUSION}

In this article, we have effectively illustrated the capacity of radar to identify life sign symptoms via the wall. The suggested procedure, which employs a shift detection algorithm, successfully identifies and locates the human's location as well as periodic breathing and pulse motion. The findings of the experiment demonstrate that the breathing and pulse speeds detected are in line with nominal values for a safe individual. Our process is highly accurate and simple to use. This technique can quickly be applied to find survivors trapped under disaster debris and other related uses.

\section{REFERENCES}

[1] D. D. Ferris, and N. C. Currie, "Survey of current technologies for through-the-wall surveillance (TWS)," Proc. SPIE, 1999, vol. 3577, pp. 62-72, doi: 10.1117/12.336988.

[2] S. I. Ivashov, V. V. Razevig, A. P. Sheyko, and I. A. Vasilyev, "Detection of human breathing and heartbeat by remote radar," In Progress in Electromagnetic Research Symposium, 2004, pp. 663-666.

[3] T. Pardhu and V. Kumar, "Reduction of Clutter using TWI Ultra wideband Imaging," Int. J. Ultra Wideband Communication and Systems, vol. 3, no. 2, pp. 101-106, 2015.

[4] E. T. Rosenbury, "Handheld instrument capable of measuring heartbeat and breathing motion at a distance," U.S. Patent, 2011.

[5] J. Li, Z. Zeng, J. Sun, and F. Liu, "Through-Wall Detection of Huamn being's Movents by UWB RADAR," IEEE Geo Science and Remote Sensing Letters, vol. 9, no. 6, 2012, doi: 10.1109/LGRS.2012.2190707.

[6] T. Pardhu and V. Kumar, "An Investigation of Human Identification Behind the wall," Jour od Adv Research in Dynamical \& Control Systems, vol. 10, no. 5, pp. 122-129, 2018.

[7] T. Pardhu, and V. Kumar, "Implementation of TWI using UWB RADAR Signals," In Proceedings of International Conference on Applications of Soft Computing Techniques in Engineering \& Technology, 2016, doi: $10.1049 / \mathrm{cp} .2016 .1496$.

[8] L. M. H. Ulander et al., "Change detection of vehicle-sized targets in forest concealment using VHF-and UHFband SAR," IEEE Aerospace and Electronic Systems Magazine, vol. 26, no. 7, pp. 30-36, 2011, doi: 10.1109/RADAR.2010.5494463.

[9] P. Runkle, L. H. Nguyen, J. H. McClellan, and L. Carin, "Multi-aspect target detection for SAR imagery using hidden Markov models," IEEE Transactions on Geoscience and Remote Sensing, vol. 39, no. 1, pp. 46-55, 2001, doi: $10.1109 / 36.898664$.

[10] M. A. Gonzalez-Huici and F. Giovanneschi, "A combined strategy for landmine detection and identification using synthetic GPR responses,” Journal of Applied Geophysics, vol. 99, 2013, doi: 10.1016/j.jappgeo.2013.08.006.

[11] F. Abujarad, A. Jostingmeier, and A. S. Omar, "Clutter Removal for Landmine using Different Signal Processing Techniques," In Proceedings of the Tenth International Conference on Grounds Penetrating Radar, 2004.

[12] X. Zhang, X. Xi, M. Li, and D. Wu, "Comparison of Impulse Radar and Spread-Spectrum Radar in Through-Wall Imaging," IEEE Transactions on Microwave Theory and Techniques, vol. 64, no. 3, pp. 699-706, Mar. 2016, doi: 10.1109/TMTT.2015.2508791.

[13] A. N. Gaikwad, D. Singh, and M. J. Nigam, "Application of clutter reduction techniques for detection of metallic and low dielectric target behind the brick wall by stepped frequency continuous wave radar in ultra-wideband range," IET Radar, Sonar \& Navigation, vol. 5, no. 4, pp. 416-425, 2011, doi: 10.1049/iet-rsn.2010.0059.

[14] L. F. Lu, M. L. Huang, Y. W. Chen, J. Liang, and Q. V. Nguyen, "Clutter Reduction in Multi-dimensional Visualization of Incomplete Data Using Sugiyama Algorithm," In 2012 16th International Conference on Information Visualisation, 2012, pp. 93-99, doi: 10.1109/IV.2012.26. 
[15] F. Brigui, G. Ginolhac, L. Thirion-Lefevre, and P. Forster, "New SAR target imaging algorithm based on oblique projection for clutter reduction," IEEE Transactions on Aerospace and Electronic Systems, vol. 50, no. 2, pp. 1118-1137, Apr. 2014, doi: 10.1109/TAES.2014.110287.

[16] Q. Cao, G. Zhang, R. D. Palmer, M. Knight, R. May, and R. J. Stafford, "Spectrum-Time Estimation and Processing (STEP) for Improving Weather Radar Data Quality," IEEE Transactions on Geoscience and Remote Sensing, vol. 50, no. 11, pp. 4670-4683, Nov. 2012, doi: 10.1109/TGRS.2012.2190608.

[17] S. Schulz, M. Becker, M. R. Groseclose, S. Schadt, and C. Hopf, "Advanced MALDI mass spectrometry imaging in pharmaceutical research and drug development," Curr. Opin. Biotechnol., vol. 55, pp. 51-59, 2019, doi: 10.1016/j.copbio.2018.08.00.

[18] M. Kebe, R. Gadhafi, B. Mohammad, M. Sanduleanu, H. Saleh, and M. Al-Qutayri, "Human Vital Signs Detection Methods and Potential Using Radars: A Review," Sensors, vol. 20, no. 5, 2020, doi: 10.3390/s20051454.

[19] S. D. Liang, "Sense-through-wall human detection based on UWB radar sensors," Signal Process, vol. 126, pp. 117-124, 2016, doi: 10.1016/j.sigpro.2015.09.022.

[20] C. Gu, and C. Li, "Assessment of human respiration patterns via noncontact sensing using Doppler multi-radar system," Sensors, vol. 15, no. 3, pp. 6383-6398, 2015, doi: 10.3390/s150306383.

[21] A. Lazaro, D. Girbau, and R. Villarino, "Techniques for clutter suppression in the presence of body movements during the detection of respiratory activity through UWB radars," Sensors, vol. 14, 2014, doi: 10.3390/s140202595.

[22] L. Chuantao et al., "A method for remotely sensing vital signs of human subjects outdoors," Sensors, vol 15, no. 7, pp. 14830-14844, 2015, doi: 10.3390/s150714830.

[23] Z. Duan and J. Liang, "Non-Contact Detection of Vital Signs Using a UWB Radar Sensor," IEEE Access, vol. 7, pp. 36888-36895, 2019, doi: 10.1109/ACCESS.2018.2886825.

[24] X. Liang, T. Lv, H. Zhang, Y. Gao, and G. Fang, "Through-wall human being detection using UWB impulse radar," J Wireless Com Network, vol. 46, 2018, doi: 10.1186/s13638-018-1054-0.

[25] S. Yoo, S. Chung, D. Seol, and S. H. Cho, "Adaptive Clutter Suppression Algorithm for Detection and Positioning using IR-UWB Radar," In 2018 9th International Conference on Ultrawideband and Ultrashort Impulse Signals (UWBUSIS), 2018, pp. 40-43, doi: 10.1109/UWBUSIS.2018.8520164.

\section{BIOGRAPHIES OF AUTHORS}

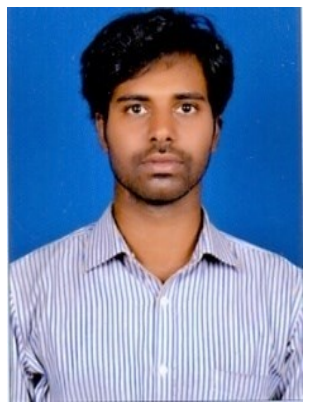

Pardhu Thottempudi became a member (M) of IEEE in 2015. Pardhu was born in Luxettipet village in Adilabad district in Telangana state, India. He completed batchelor's degree B.tech in the stream of electronics and communication engineering in 2011 from MLR Institute of Technology, Hyderabad, India. He has done his master's degree M.Tech in embedded systems from Vignan's University, Vadlamudi in 2013. He is pursuing Ph.D in the stream of radar signal processing in VIT University. His major fields of interests include digital signal processing, radar communications, embedded systems, and implementation of signal processing on applications in FPGA. He is working as assistant professor of department of electronics and communication engineering in MLR institute of technology, Hyderabad, India since 2016. He also worked as project intern in research centre imarat, Hyderabad. He published 28 research papers on VLSI, image processing, antennas, signal processing, radar communications in reputed international journals and various IEEE conferences. Pardhu Thottempudi is the life member of ISTE, associate member of IETE from 2015. He is the reviewer of many international reputed journals like WSEAS transactions, AIRCCSE.

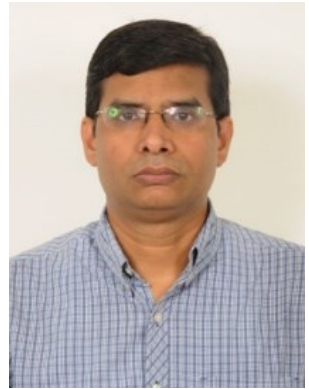

Vijay Kumar received M. Sc electronics from the Magadh University, India in 2003. He has completed M.Tech degree in remote sensing with specialization in microwave sensing techniques from BIT Mesra, Ranchi, in 2005 and completed Ph. D from IIT Bombay, Mumbai in 2011. DST young Scientist project award has been given to him in 2009 and worked as PI and Co-PI for the projects sponsored by DST, and german aerospace agency (DLR). He worked as post-doctoral fellow and DST sponsored young scientist at IIT Bombay during Feb. 2011 to Jan. 2013. Presently, Kumar is working as associate professor at School of Electronics, VIT University, Vellore TN, India. He has been author and co-author of many peer reviewed journal paper and more than thirty IEEE proceeding papers. Kumar's research interests are in SAR processing algorithm development, InSAR techniques, and SAR image processing and pattern recognition and radar antenna designing. He is a life member of ISRS, member of AGU, SPIE and IEEE. He has been reviewer of journals SPIE JARS, IEEE geosciences and remote sensing, ISRS. 